\title{
Understanding the "anti-kick" in the merger of binary black holes
}

\author{
Luciano Rezzolla, ${ }^{1,2}$ Rodrigo P. Macedo, ${ }^{1,3}$ and José Luis Jaramillo ${ }^{1,4}$ \\ ${ }^{1}$ Max-Planck-Institut für Gravitationsphysik, Albert Einstein Institut, Potsdam, Germany \\ ${ }^{2}$ Department of Physics and Astronomy, Louisiana State University, Baton Rouge, Louisiana, USA \\ ${ }^{3}$ Instituto de Física, Universidade de São Paulo, São Paulo, SP, Brazil \\ ${ }^{4}$ Laboratoire Univers et Théories, Observatoire de Paris, CNRS, Meudon, France
}

\begin{abstract}
The generation of a large recoil velocity from the inspiral and merger of binary black holes represents one of the most exciting results of numerical-relativity calculations. While many aspects of this process have been investigated and explained, the "antikick", namely the sudden deceleration after the merger, has not yet found a simple explanation. We show that the antikick can be understood in terms of the radiation from a deformed black hole where the anisotropic curvature distribution on the horizon correlates with the direction and intensity of the recoil. Our analysis is focussed on Robinson-Trautman spacetimes and allows us to measure both the energies and momenta radiated in a gauge-invariant manner. At the same time, this simpler setup provides the qualitative and quantitative features of merging black holes, opening the way to a deeper understanding of the nonlinear dynamics of black-hole spacetimes.
\end{abstract}

PACS numbers: 04.30.Db, 04.25.dg, 04.70.Bw, 97.60.Lf

Introduction. The merger of two black holes (BHs) is one of the most important sources of gravitational waves (GW) and it is generally accompanied by the recoil of the final $\mathrm{BH}$ as a result of anisotropic GW emission. While this scenario has been investigated for decades [1] and first estimates have been made using approximated and semianalytical methods such as a particle approximation [2], post-Newtonian methods [3] and the close-limit approximation (CLA) [4], it is only recently that accurate values for the recoil have been computed [5-12].

Besides being a genuine nonlinear effect of general relativity, the generation of a large recoil velocity during the merger of two BHs has a direct impact in astrophysics. Depending on its size and its variation with the mass ratio and spin, in fact, it can play an important role in the growth of supermassive BHs via mergers of galaxies and on the number of galaxies containing BHs [13]. Numerical-relativity simulations of BHs inspiralling on quasicircular orbits have already revealed many of the most important features of this process showing, for instance, that asymmetries in the mass can lead to recoil velocities $v_{\mathrm{k}} \lesssim 175 \mathrm{~km} / \mathrm{s}$ [5, 6], while asymmetries in the spins can lead respectively to $v_{\mathrm{k}} \lesssim 450 \mathrm{~km} / \mathrm{s}$ or $v_{\mathrm{k}} \lesssim 4000 \mathrm{~km} / \mathrm{s}$ if the spins are aligned $[8,[9,11]$ or perpendicular to the orbital angular momentum [7, 14, 15] (see [16] for a review).

At the same time, however, there are a number of aspects of the nonlinear processes leading to the recoil that are far from being clarified even though interesting work has been recently carried out to investigate such aspects [17-19]. One of these features, and possibly the most puzzling one, is the generic presence of an "antikick", namely, of one (or more) decelerations experienced by the recoiling $\mathrm{BH}$. Such antikicks take place after a single apparent horizon $(\mathrm{AH})$ has been found and have been reported in essentially all of the mergers simulated so far.

This Letter is dedicated to elucidate the stages during which the antikick is generated and to provide a simple and qualitative interpretation of the physics underlying this process. Our focus will be on the head-on collision of two nonspinning BHs with different mass and although this is the simplest scenario for a $\mathrm{BH}$-merger, it contains many of the aspects that can be encountered in more generic conditions. Our qualitative picture will then be made quantitative and gauge-invariant by studying the logical equivalent of this process in the evolution of a Robinson-Trautman (RT) spacetime, with measurements of the recoil made at future null infinity. As commented below, the insight gained with RT spacetimes will be valuable to explain the antikick under generic conditions.

The basic picture. Before discussing how to use the RT spacetime to compute the antikick, it is useful to illustrate the basic $\mathrm{BH}$ physics leading to such process and for this we consider the collinear merger of two Schwarzschild BHs with unequal masses. This is shown in a schematic cartoon in Fig. 1, where we have considered a reference frame in the centre of mass of the system and where the smaller black hole is initially on the positive $z$ axis, while the larger one is on the negative axis. As the two BHs free-fall towards each other, the smaller one will move faster and will be more efficient in "forwardbeaming" its GW emission [3]. As a result, the linear momentum will be radiated mostly downwards, thus leading to an upwards recoil of the BH binary [ $c f$. stage (1) in Fig.1]. At the merger the $\mathrm{BH}$ velocities will be larger and so will also be the anisotropic GW emission and the corresponding recoil of the system. However, when a single $\mathrm{AH}$ is formed comprising the two BHs, the curvature distribution on this 2-surface will be highly anisotropic, being higher in the upper hemisphere [cf. shading in stage (2) of Fig. 1]. Because the newly formed $\mathrm{BH}$ will want to radiate all of its deviations away from the final Schwarzschild configuration, it will do so more effectively there where the curvature is larger, thus with a stronger emission of GWs from the northern hemisphere. As a result, after the merger the linear momentum will be emitted mostly upwards and this sudden sign change will lead to the antikick. The anisotropic GW emission will decay exponentially as the curvature gradients are erased and the $\mathrm{BH}$ will have reached its final and decelerated recoil velocity [ $c f$. stage (3)].

Although this picture refers to a head-on collision, it is supported by the findings in the CLA (where the direction of the ringdown kick is approximately opposite to that of the accumulated inspiral plus plunge kick) [17] and it can be gener- 


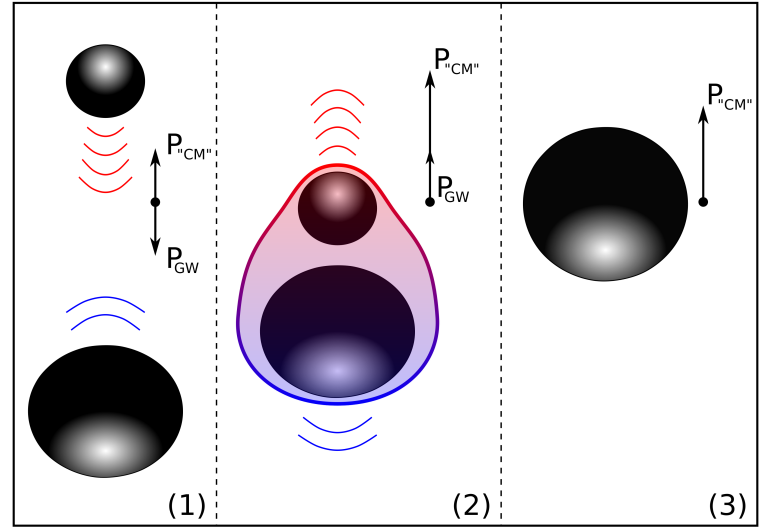

FIG. 1: Cartoon of the generation of the antikick in the head-on collision of two unequal-mass Schwarzschild BHs. Initially the smaller $\mathrm{BH}$ moves faster and linear momentum is radiated mostly downwards, thus leading to an upwards recoil of the system [stage (1)]. At the merger the curvature is higher in the upper hemisphere of the distorted $\mathrm{BH}$ ( $c f$. shading) and linear momentum is radiated mostly upwards leading to the antikick [stage (2)]. The BH decelerates till a uniform curvature is restored on the horizon [stage (3)].

alized to a situation in which the BHs have different masses, different spins and are merging through an inspiral. Also in a more generic case, in fact, the newly formed $\mathrm{AH}$ will have a complicated but globally anisotropic distribution of the curvature, determining the direction (which is in general varying in time) along which the GWs will be emitted. Therefore we argue that the geometric properties in a dynamical horizon (of a black or white hole) determine its global dynamics. We next use the RT spacetime to validate this picture.

The Robinson-Trautman spacetime. It is a class of vacuum solutions admitting a congruence of null geodesics which are twist and shear-free [20], with a future stationary horizon and a dynamical past (outer trapping) horizon [21] (past AH hereafter). A RT spacetime can thus be regarded as an isolated nonspherical white hole emitting GWs, where the evolution of the AH curvature-anisotropies and of the spacetime momentum can be related unambiguously. The metric is [22]

$d s^{2}=-\left(K-\frac{2 M_{\infty}}{r}-\frac{2 r \partial_{u} Q}{Q}\right) d u^{2}-2 d u d r+\frac{r^{2}}{Q^{2}} d \Omega^{2}$,

where $Q=Q(u, \Omega), u$ is the standard null coordinate, $r$ is the affine parameter of the outgoing null geodesics, and $\Omega=\{\theta, \phi\}$ are the angular coordinates on the unit sphere $S^{2}$. Here $M_{\infty}$ is a constant and is related to the asymptotic mass, while the function $K(u, \Omega)$ is the Gaussian curvature of the surface corresponding to $r=1$ and $u=$ constant, $K(u, \Omega) \equiv Q^{2}\left(1+\nabla_{\Omega}^{2} \ln Q\right)$, where $\nabla_{\Omega}^{2}$ is the Laplacian on $S^{2}$. The Einstein equations then lead to

$$
\partial_{u} Q(u, \Omega)=-Q^{3} \nabla_{\Omega}^{2} K(u, \Omega) /\left(12 M_{\infty}\right) .
$$

Any regular initial data $Q=Q(0, \Omega)$ will smoothly evolve according to (2) until it achieves a stationary configuration corresponding to a Schwarzschild $\mathrm{BH}$ at rest or moving with a constant speed [23]. Equation (2) implies the existence of the constant of motion $\mathcal{A} \equiv \int_{S^{2}} d \Omega / Q^{2}$, which clearly represents the area of the surface $u, r=$ constant and can be used to normalise $Q$ so that $\mathcal{A}=4 \pi$. All the physically relevant information is contained in the function $Q(u, \Omega)$, and this includes the gravitational radiation, which can be extracted by relating $Q(u, \Omega)$ to the radiative part of the Riemann tensor [24, 25].

The past AH radius $R(u, \Omega)$ is given by the vanishing expansion of the future ingoing null geodesics [21]

$$
Q^{2} \nabla_{\Omega}^{2} \ln R=K-2 M_{\infty} / R .
$$

The mass and momentum of the $\mathrm{BH}$ are computed at future null infinity using the Bondi 4-momentum [22]

$$
P^{\alpha}(u) \equiv \frac{M_{\infty}}{4 \pi} \int_{S^{2}} \frac{\eta^{\alpha}}{Q^{3}} d \Omega,
$$

with $\left\{\eta^{\alpha}\right\}=\{1, \sin \theta \cos \phi, \sin \theta \sin \phi, \cos \theta\}$. Given smooth initial data, the spacetime will evolve to a stationary nonradiative solution which, in axisymmetry, has the form $Q(\infty, \theta)=$ $(1 \mp v x) / \sqrt{1-v^{2}}$, with $x \equiv \cos \theta$ [22]. The Bondi 4momentum associated to $Q(\infty, \theta)$ is

$$
\{P(\infty)\}^{\alpha}=\left(M_{\infty} / \sqrt{1-v^{2}}\right)\{1,0,0, \pm v\},
$$

so that the parameter $v$ in $Q(\infty, \theta)$ can be interpreted as the velocity of the Schwarzschild BH in the $z$-direction.

One of the difficulties with RT spacetimes is the definition of physically meaningful initial data. Although we are more interested in a proof of principle than in a realistic configuration, we have adopted the prescription in [25]

$$
Q(0, \theta)=Q_{0}\left[\frac{1}{\sqrt{1-w x}}+\frac{q}{\sqrt{1+w x}}\right]^{-2},
$$

which was interpreted to represent the final stages (i.e., after a common $\mathrm{AH}$ is formed) of a head-on collision of two boosted BHs with opposite velocities $w$ and mass ratio $q[25]$. In practice, to reproduce the situation shown in Fig. 11 we have set $w<0$ and taken $q \in[0,1]$, but a more general class of initial data can be easily constructed. Note that $Q_{0}$ is normalized so that to $\mathcal{A}=4 \pi$ and that in general the deformed $\mathrm{BH}$ will not be initially at rest. As a result, given the initial velocity $v_{0} \equiv P^{3}(0) / P^{0}(0)$, we perform a boost $\bar{P}^{\alpha}=\Lambda_{\beta}^{\alpha}\left(v_{0}\right) P^{\beta}$ so that $\bar{P}^{3}(0)=0$ by construction. The numerical solution of Eq. (2) with initial data (6) is performed as discussed in [22]. Discussion. Figure 2 reports the typical evolution of a RT spacetime with the lower panel showing the evolution of the curvature of the past $\mathrm{AH} K_{\mathrm{AH}} \equiv 2 M_{\infty} / R^{3}(x)$ at the north $(x=1)$ and south pole $(x=-1)$, and with the upper panel showing the evolution of the recoil velocity. Note that the two local curvatures are different initially, with the one in the upper hemisphere being larger than the one in the lower hemisphere ( $c f$. Figure 11). However, as the gravitational radiation is emitted, this difference is erased. When this happens, the deceleration stops and the $\mathrm{BH}$ attains its asymptotic recoil velocity. The inset reports the curvature difference relative to the asymptotic Schwarzschild one, $K_{\mathrm{AH}}-1$, whose exponentially decaying behaviour is the one expected in a ringing $\mathrm{BH}$. 


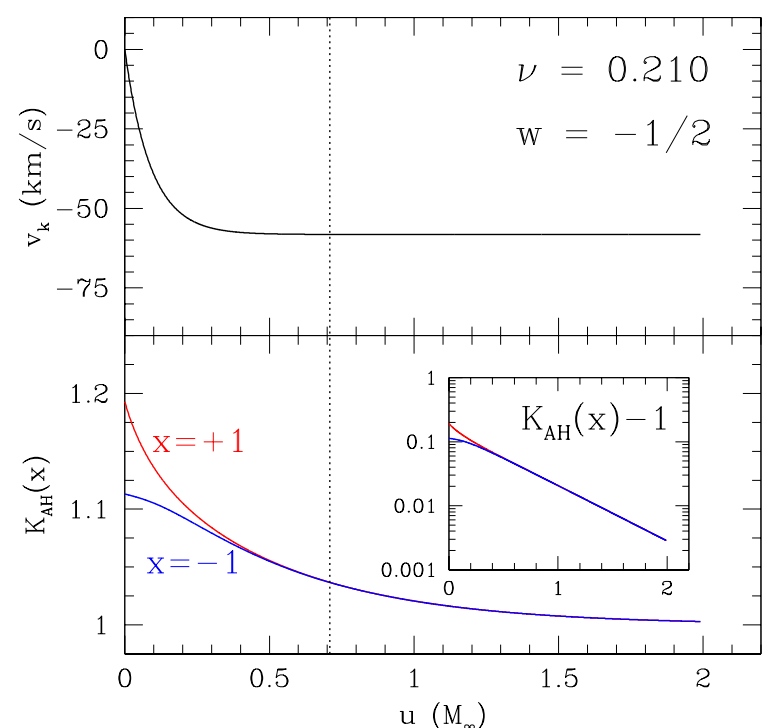

FIG. 2: Typical evolution of a RT spacetime. Shown in the lower panel is the evolution of the curvature $K_{\mathrm{AH}}$ at the north $(x=1)$ and south pole $(x=-1)$. Shown in the upper panel is the evolution of the recoil, which stops decreasing when the curvature difference is erased by the radiation (dotted line).

As mentioned before, that shown in Fig.2 is a typical evolution of a RT spacetime and is not specific of the initial data (6). By varying the values of $w$, in fact, it is possible to increase or decrease the final recoil, while a sign change in $w$ simply inverts the curvature at the poles so that, for instance, initial data with $w>0$ would yield a $\mathrm{BH}$ accelerating in the positive $z$-direction. Interestingly, it is even possible to fine-tune the parameter $w$ so that the recoil produced for a RT spacetime mimics the antikick produced by the quasicircular inspiral of nonspinning binaries. This is shown in Fig. 3, which reports the recoil as a function of the symmetric mass ratio $\nu \equiv q /(1+q)^{2}$, and where the dashed line refers to the antikick for the inspiral of nonspinning binaries in the CLA [17] (the parameters chosen, i.e., $w=-0.425$ and $r_{12}=2 M$, are those minimizing the differences). Considering that the two curves are related only logically and that the CLA one contains all the information about inspiralling BHs, including the orbital rotation, the match is surprisingly good.

It is also suggestive to think that the curve in Fig. 3 is actually composed of two different branches, one of which is characterized by large curvature gradients across the AH but small values of the curvature (this is the low- $\nu$ branch and is indicated with squares), while the other is characterized by small curvature gradients and large values of the curvature (this is the high- $\nu$ branch and is indicated with circles). The same recoil velocity can then be produced by two different values of $\nu$, for which the effects of large curvature gradients and small curvatures are the same as those produced by small curvature gradients but large curvatures.

To go from this intuition to a mathematically well-defined measure we have computed the mass multipoles of the intrinsic curvature of the initial data using the formalism developed in [26] for dynamical horizons. Namely, we have calculated

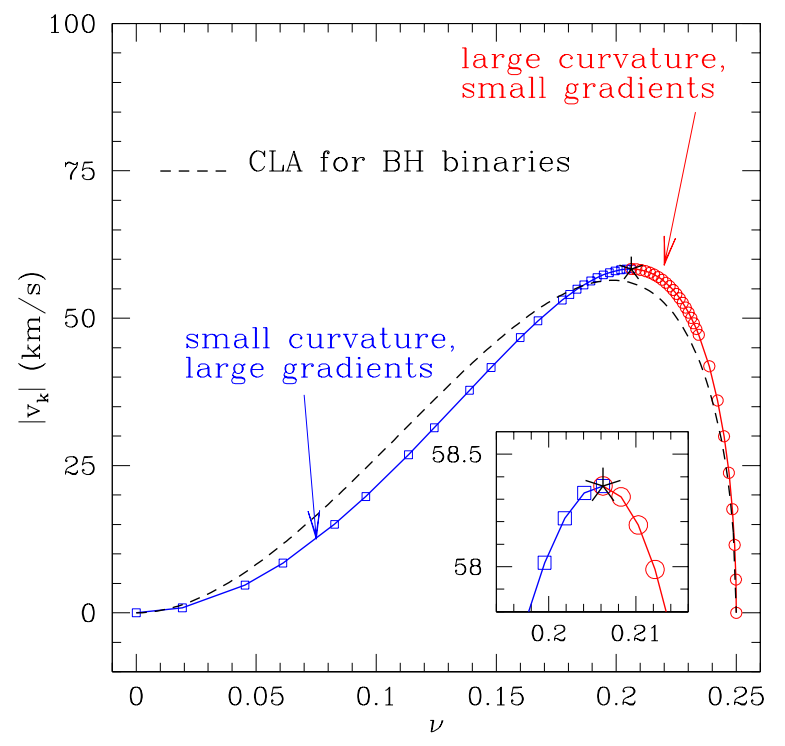

FIG. 3: Recoil velocity shown as a function of the symmetric mass ratio $\nu$ when $w=-0.425$, with the dashed line refers to the antikick from the inspiral of nonspinning binaries in the CLA [17]. Note that the curve can be thought of as being composed of two different branches.

the mass moments as

$$
M_{n} \equiv \oint \frac{P_{n}(\tilde{x})}{Q^{2}(\theta) R(\theta)} d \Omega,
$$

where $P_{n}(\tilde{x})$ is the Legendre polynomial in terms of the coordinate $\tilde{x}(\theta)$ which obeys $\partial_{\theta} \tilde{x}=-\sin \theta R(\theta)^{2} /\left(R_{\mathrm{AH}}^{2} Q(\theta)^{2}\right)$, with $R_{\mathrm{AH}} \equiv \sqrt{\mathcal{A}_{\mathrm{AH}} /(4 \pi)}$ and $\tilde{x}(0)=1$. Using these multipoles it is possible to construct an effective-curvature parameter $K_{\text {eff }}$ that represents a measure of the global curvature properties of the initial data and from which the recoil depends in an injective way. Because this effectivecurvature parameter has to contain the contribution from the even and odd multipoles, we have found that the expression $K_{\text {eff }}=M_{2}\left|\sum_{n=1} M_{2 n+1} / 3^{n-1}\right|$, reproduces exactly what is expected (note $M_{1}=0$ to machine precision).

This is shown in Fig. 4 which reports the recoil velocity as a function of $K_{\text {eff }}$. As predicted, and in contrast with Fig. 3, the relation between the curvature and the recoil is now injective, with the maximum recoil velocity being given by the maximum value of $K_{\text {eff }}$ (see inset), and with the two branches coinciding. We do not expect the expression found here for $K_{\text {eff }}$ to be unique and indeed a more generic one will have to include also the mass-current multipoles to account for the spin contributions. However, lacking a rigorous mathematical guidance, our phenomenological $K_{\text {eff }}$ is a reasonable, intuitive approximation.

Conclusions. We have outlined a simple picture to explain the deceleration observed during the merger of binary BHs in terms of the dissipation of an anisotropic distribution of curvature on the horizon of the newly formed $\mathrm{BH}$. We have analyzed this picture for the head-on collision of two nonspinning $\mathrm{BHs}$ with unequal mass but its extension to generic systems is 


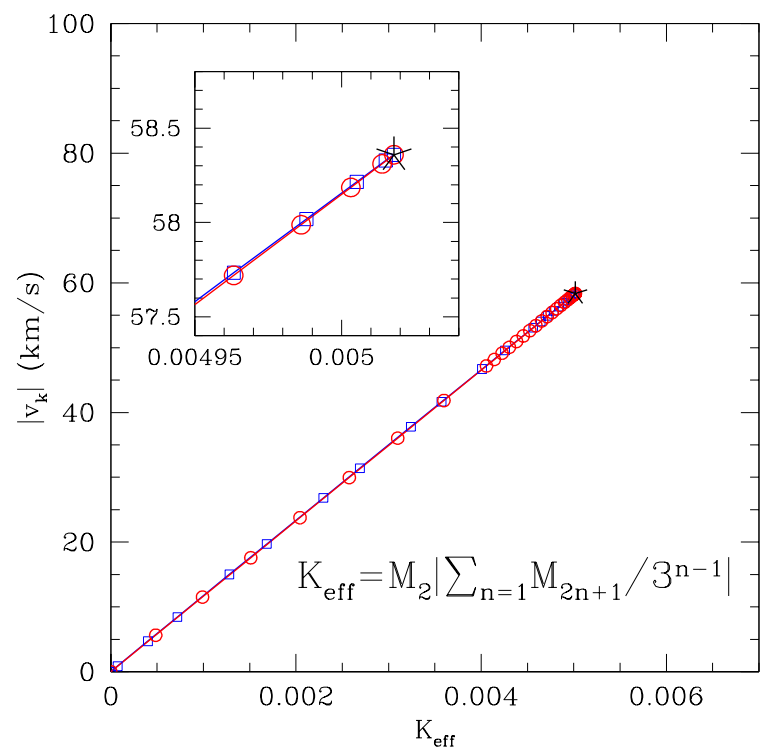

FIG. 4: Recoil velocity shown as a function of the effective curvature. In contrast with Fig. 3. which uses the same symbols employed here, the relation between the curvature and the recoil is now injective.

direct as the same features will be present also when includ- ing the spin and the orbital contributions: mass-current multipoles will add (substract) in prograde (retrograde) orbits. The qualitative arguments made on the head-on collision have then been made quantitative by analyzing the gauge-independent dynamics of RT spacetimes. More specifically we have shown that the deceleration is associated to the radiation of curvature differences and persists as long as the gradients are not erased. Furthermore, the directionality of the recoil is dictated by the north-south curvature gradients and a one-to-one mapping between the recoil and an effective curvature is possible. These results presented here can help in understanding some nonlinear aspects of curved spacetimes.

Finally, an alternative interpretation of the recoil phenomenology can be given via the Landau-Lifshitz pseudotensor, where the recoil is given by the cancellation of large and opposite fluxes of momentum, part of which are "swallowed" by the $\mathrm{BH}$ [27]. While this is an interesting route, it relies on gauge-dependent measurements which may themselves be counterintuitive.

Acknowledgments. It is a pleasure to thank A. Saa, B. Krishnan, H. Oliveira, B. Schutz, and I. Soares for useful discussions. We are also grateful to A. Le Tiec for providing his estimates of the recoil in the CLA. This work was supported in part by the DAAD and the DFG grant SFB/Transregio 7.
[1] A. Peres, Phys. Rev. 128, 2471 (1962); J. D. Bekenstein, Astrophys. J. 183, 657 (1973).

[2] M. J. Fitchett and S. Detweiler, Mon. Not. R. astr. Soc. 211, 933 (1984); T. Nakamura and M. P. Haugan, Astrophys. J. 269, 292 (1983); M. Favata, S. A. Hughes, and D. E. Holz, Astrophys. J. 607, L5 (2004).

[3] A. G. Wiseman, Phys. Rev. D 46, 1517 (1992); L. E. Kidder, Phys. Rev. D 52, 821 (1995); L. Blanchet, M. S. S. Qusailah, and C. M. Will, Astrophys. J. 635, 508 (2005); T. Damour and A. Gopakumar, Phys. Rev. D 73, 124006 (2006).

[4] Z. Andrade and R. H. Price, Phys. Rev. D 56, 6336 (1997); C. F. Sopuerta, N. Yunes, and P. Laguna, Phys. Rev. D 74, 124010 (2006).

[5] J. G. Baker, et al. Astrophys. J. 653, L93 (2006).

[6] J. A. Gonzalez, et al. Phys. Rev. Lett. 98, 091101 (2007).

[7] M. Campanelli, et al. Phys. Rev. Lett. 98, 231102 (2007).

[8] F. Herrmann, et al. Astrophys. J. 661, 430 (2007).

[9] M. Koppitz et al., Phys. Rev. Lett. 99, 041102 (2007).

[10] C. O. Lousto and Y. Zlochower, Phys. Rev. D 77, 044028 (2008).

[11] D. Pollney et al., Phys. Rev. D 76, 124002 (2007).

[12] J. Healy et al., Phys. Rev. Lett. 102, 041101 (2009).

[13] A. Gualandris and D. Merritt Astrophys. J 678, 780 (2008); S. Komossa and D. Merritt, Astrophys. J. 683, L21 (2008).

[14] M. Campanelli, C. O. Lousto, Y. Zlochower, and D. Merritt, Astrophys. J. 659, L5 (2007);
[15] J. A. Gonzalez, et al. Phys. Rev. Lett. 98, 231101 (2007).

[16] L. Rezzolla, Class. Quant. Grav. 26, 094023 (2009).

[17] A. Le Tiec, L. Blanchet, and C. M. Will, Class. Quant. Grav. 27, 012001 (2010); A. Le Tiec, L. Blanchet, Class. Quant. Grav. 27, 045008, (2010).

[18] J. D. Schnittman, et al. Phys. Rev. D 77, 044031 (2008).

[19] Y. Mino and J. Brink, Phys. Rev. D 78, 124015 (2008).

[20] I. Robinson and A. Trautman, Proc. Roy. Soc. Lond. A 265, 463 (1962)

[21] R. Penrose, Ann. NY Acad. Sci., 224, 115 (1973); K. P. Tod, Class. Quantum Grav. 6, 1159 (1989); E. W. Chow and A. W. Lun, J. Aust. Math. Soc. B 41, 217 (1999); W. Natorf and J. Tafel, Class. Quant. Grav. 25, 195012 (2008); J. Podolský and O. Svítek, Phys. Rev. D 80, 124042 (2009).

[22] R. P. Macedo and A. Saa, Phys. Rev. D 78, 104025 (2008).

[23] P. Chrusciel, Commun. Math. Phys. 137, 289 (1991).

[24] H. P. de Oliveira and I. Damiao Soares, Phys. Rev. D 70, 084041 (2004); H. P. de Oliveira and E. L. Rodrigues, Class. Quant. Grav. 25, 205020 (2008).

[25] R. F. Aranha, H. P. de Oliveira, I. Damiao Soares, and E. V. Tonini, Int. J. Mod. Phys. D 17, 2049 (2008).

[26] A. Ashtekar, J. Engle, T. Pawlowski, and C. Van Den Broeck, Class. Quantum Grav. 21, 2549 (2004); E. Schnetter, B. Krishnan, and F. Beyer, Phys. Rev. D 74, 024028 (2006).

[27] G. Lovelace et al. (2009), 0907.0869. 